Journal of Computer Science 3 (3): 122-129, 2007

ISSN 1549-3636

(C) 2007 Science Publications

\title{
Users Assistants for E-Learning Environment Over the Web
}

\author{
${ }^{1}$ Lamri Douidi, ${ }^{2}$ Mahieddine Djoudi and ${ }^{1}$ Chabane Khentout \\ ${ }^{1}$ Department of computer science, University Ferhat Abbas of Setif, Algeria \\ ${ }^{2}$ Laboratoire SIC et équipe IRMA, UFR Sciences SP2MI Université de Poitiers Téléport 2 \\ BP 3017986960 Futuroscope Cedex, France
}

\begin{abstract}
In this study, we present users assistants for e-learning environment over the Web called AVUNET. It was made up of an educational server that allows access to the available courses on the site. The server was structured in pedagogical labs that respond to the user needs. It offers also a module for self-evaluation so that the user can evaluate his/her level. Trainers have created this facility very carefully to assure a detailed evaluation with very accurate solutions. Based on the model of "telephone ring", the system proposes facilities for communication and collaboration in order to bring the trainers and the learners closer to each other. This forum of trainers and learners allow the users to exchange information and their experiences pedagogic. These experiences are acquired by the use of computer aided tools for virtual navigation through structured hypertext documents. The user actions during a learning or navigation session are analyzed.
\end{abstract}

Key words: Authoring system, e-learning, content design, learner assistance

\section{INTRODUCTION}

Among the potential of the advent of communication and information technologies, in the field of the e-learning, is the development of environments that support distance co-operative training. Within this framework, we propose an experimental platform of a co-operative system. It gives the possibility to a group of users (learners, teachers and tutors) to cooperate, to coordinate and to communicate in order to arrive to a better form of training.

Overview of avunet environment: AVUNET (Algerian Virtual University) is a multilanguage environment for distance education making use of the new information and communication technology specifically Internet and hypermedia. It has a structure close to that of LearningSpace, TopClass, Librarian or WebCT. Based on client-server architecture, the platform is developed in PHP/MySQL and is software independent. The data set is stored on the server in a centralized database ${ }^{[1,2]}$.

Platform addresses users, who are either at the university, home or connected from access points and aspire to be trained, supplement their knowledge or to evaluate their qualification levels. The Information module presents a detailed description of the platform and the access methods and use with level and prerequisites indication.

AVUNET environment contains three systems ${ }^{[1,2]}$.

1. A communication and management server made up of several modules. An information module which contains the various files and data needed by the user's teaching or training activities. A co-operative and communication module which has the means to make it possible for users to interact with each other, to accomplish team works or to take part in discussions. In order to favor the co-operative learning, the interfaces are conceived in such a way to make the presence of the others known by providing indication of their availability and their remarks on the teaching material.

2. A teacher assistants contains the necessary tools for tasks' production. It contains amongst other things a content design environment and an evaluation space to improve the learner knowledge and abilities.

3. A learner system which makes it possible for the learner to obtain assistance or advise or an adaptation of the environment from the computer system. The objective of the designed system is to give the learner the possibility to locate $\mathrm{him} /$ herself with respect to time and space during a training session. The learner is presented with a chart of courses and visited pages, thus enabling him/her to have an explicit representation of the virtual space. Various visualization levels are set up in order to make the chart more visible and not overloaded. A temporal panel is displayed permanently allowing the learner to monitor and optimize the training time. The user has also the possibility to access online help and a glossary containing the terms frequently found on Internet and likely to be misunderstood by beginner. The system gives access to a set of tools: notebook, diary, work plan, $\operatorname{etc}^{[3]}$.

Corresponding Author: Lamri Douidi, Département d'informatique, Université Ferhat Abbas, 19000 Sétif, Algérie 


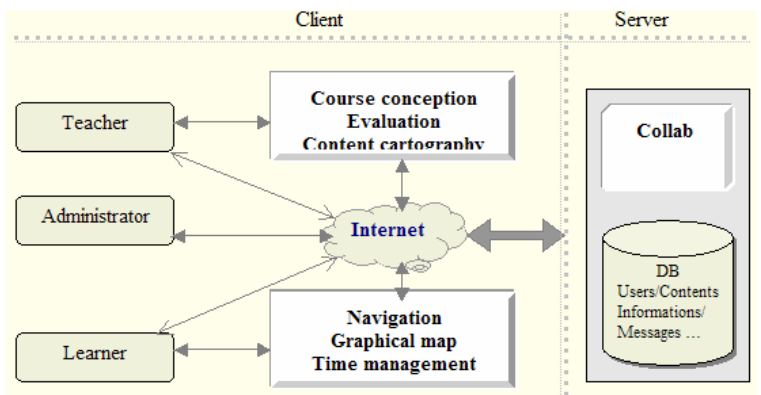

Fig. 1: AVUNET architecture

The efficiency of an educational site is mainly based on the way textual as well as graphical information is organized, on the navigation flexibility and interactivity. Consequently, we have elaborated a design guide according to our appraisal concerning sites building and taking into account several web sites analyses. Such guide is intended to facilitate teachers' task, while enabling them to build cognitive courses through a design plan and recommendations.

A course building serves to structure its content in order to facilitate its training, what will allows to reach pedagogical objectives and significantly reduce problems raised by users. Such design process may be achieved following several steps. First, it is necessary to identify specific training objectives and structure content into logical training units. Second, a complete use scenario of use of the web site has to be achieved. Finally, navigation flow chart and site logical links have to be specified, allowing thus user to build his own knowledge mental structure and then produce pages models. A model is intended to standardize semantic units' presentation of the web site (typography, page setup, title, graphical element locations, etc.).The educative server essentially focuses on information structuring and organization according to learner's abilities and skills, which are aimed at learner model building. It provides to learners structured courses that are linked to several related exercises. Distance on-line courses are aimed at familiarizing learners with basic concepts of communication and networking and providing them with new ways of teaching and learning using distance teaching techniques. Furthermore, several collaborative tools have been designed to give them an opportunity to work together.

The server is structured into educational workshops, whose contents are individually or collectively built by teachers. Based on virtual book metaphor, every course is structured as chapters, sections and paragraphs. A course has a modular structure, including a presentation page, which contains links to a table of contents, glossary words and an on line bibliographic list. Navigation within course components is achieved using buttons and arrows.

For a start, students must execute a formal registration that should be transmitted to the server for authentication and options selecting (i.e. novice or expert mode, sound, etc.). After finishing the course, a student will gain experience and may study the subject thoroughly (under expert mode) or evaluate his knowledge according to several parameters specification. This will help adapting training materials and interaction according to learner's performance. The underlying mechanisms are Java interface and interactive HTML forms. Such forms are automatically transmitted to the server and will be processed. After which, another page of information is returned to the learner for review. Thus, learners are enabled to communicate with teachers through email and from any web page, they may ask a question or be well informed about frequently asked questions as well as the associated answers of teachers.

Communication model: Communication process is achieved through the interaction of three elements: a message sender, a channel and a receiver. This process can be more or less complex according to the number of people implied and the broadcast technique used. Communication within a group requires for a participant not only information retrieval from a distant source, but also to be able to quickly access it once found. The requirement for a low cost immediate storage of objects in current use brought designers to define the workspace concept. Such space constitutes an environment in which resources made available are tailored according to needs of work process that will use them. Thus, exploring real world metaphors as well as graphical facilities allows us to achieve a considerable advance for man-machine interfaces area and build more complex interaction models such as those required for group activities.

However, despite the availability of several communications models, their ability to enhance group work productivity among several users have not been proved. In fact, our studies on the subject have led us to the conclusion that building efficient communication models does not represent a technical prowess. In contrast, it is necessary and important to consider the most common and usual way used by users for their communications. Consequently, using interaction metaphors extracted from users daily life may be a very likely approach to draw nearer users behavior. Furthermore, this will effectively unload users of the software-training phase. While telephone is the most fluently used tool for communication between people, it seems obvious that such issue have to be explored. So, we built a natural communication model based on a new metaphor called "Telephone Ring". The following sections are consecrated to discuss and show the interest of our approach.

Telephone ring metaphor: Telephone Ring ${ }^{[3]}$ is rather a long connection that can be interrupted. It allows establishing a communication with a correspondent; 
this one is notified by a telephone sound (and possibly by displaying the caller number). Telephone Ring is a reciprocal connection between two or several users, one is the caller and the others must give their agreement, so that the communication is enabled. Telephone Ring metaphor may be characterized by three main elements:

* Reciprocity: any user who is able to call should be called;

* Feedback: information must be returned to the called person such as caller's identity, when, etc.

* Privacy: To protect his workspace, a user may pick up the receiver, he will be then in an occupied situation and doesn't answer either and nobody may join him. Such protection may be achieved through a filtering mechanism, for example by setting on an automatic answering voice.

Communication support: The system software architecture is organized into layers around a server; communication component within process layer manages connections such as a switchboard. The component on the server top manages access control and feedback information. Such decomposition into layers is based on the separating concept of mechanism from usage strategy. Here are some concepts around which the system had been built:

* Telephone ring action may be assimilated to a collaboration request expressed by a distant user or even the server itself. While he has no call, any user can independently do his work or achieve a task for the community.

* According to recent telephone technology, we will first time suppose that one cannot call someone that is already in line. However, in next phases, we will allow user to speak in alternation with several other users and even establish a multiple connections with several other users.

* Every user process updates locally (independently from the other sites) its interface widgets (such as when someone increases the sound of his telephonic device, etc.).

Applying to training situations: Designing an efficient collaborative interaction model leans on the way interaction modes between users are selected. In other words, such modes have to be natural and without constraints. Collaborative learning process is characterized by a series of phases during which, students work individually or collectively. In the same way, to enhance knowledge and skills exchange, it is necessary to allow a learner to draw one's inspiration from more experienced people. Therefore, we defined for AVUNET three collaboration modes through which the following learning situations may be achieved:

* Star training: a teacher supervises simultaneously several students training from a remote location. He interacts individually with students, supervises personal work progressing of each one or providing assistance and advises to those whom need it. Communications among students are unauthorized and teacher doesn't deliver any common message to them. Thus, teacher has to synchronize students' work and catch up shifts between them. The main goal of such interaction mode is to increase each student's individual ability.

* Interpersonal communication: Teacher poses a same problem to all students, whom will interact with him. Teacher in such case may sometimes send a same message to the students (advice, document, problem, examples, etc.) and authorize in certain cases a communication or information exchange between learners.

* Collective problem resolution: it is a typical situation of cooperation teacher poses a problem to the whole of learners whom will cooperate to solve it. Collaboration among students may be free while all learners have a same role or regulated when students interact with a pre-established role. This interaction situation is essentially intended to increase student's ability to work within a group.

Applying a specific training situation has to be done according to the group structure. A situation analysis mechanism is foreseen to provide assistance for group members and help them to take good decisions. In addition, the system includes specific tools designed to manage shared resources, individual and collective knowledge and appropriated communication devices ${ }^{[3]}$.

\section{Teacher assistant}

Methodology: Our objective is to design and implement an authoring system for content design in a format directly accessible by the learner. It is a matter of proposing a representation model for the content related to the topic being taught, taking into account the perspectives of educational domain and pedagogic. Our idea is to create and organize an online access environment to a rich and varied content, which can support course development, planning and implementation teaching and some aspects related to learner modeling ${ }^{[4,5]}$.

Our contents design approach supports three prospects for topic organization: domain, pedagogy and didactic. In this approach the domain aspect is achieved by a model, which represents and organizes the domain knowledge based on the existing logical links. The organization of the objectives consists in particular in modeling the necessary preconditions to their realization and studying the impact that an objective can have on domain knowledge. Didactic dimension amounts to producing a model that defines and organizes the different tactical means necessary to the teaching of the considered topic.

Pedagogical and graphical chart definition: The quality of a teaching environment on the web is based 
mainly on the information (textual and/or graphic) organization, the navigation flexibility and the interactivity. The design of a teaching platform on Internet supposes the analysis of the needs and the site relevance ${ }^{[6]}$. The needs' analysis will help in answering the following questions:

* What are the needs of the educational platform: interactivity, exercises, simulations, information retrieval, data processing, etc?

* Why use the Internet: distance learning, computerassisted co-operative work, interactive learning, pedagogic advising, etc.

* Who are concerned: academicians, distance learners, training for companies, general public, etc?

* What are the technical considerations to take into account: computer power, screen type and size, multi-media, Internet network, etc?

* And finally how is the time distributed on the project.

The teaching design consists in: defining the goals and the objectives, structuring the contents, setting the teaching strategies, designing the interface parameters, building the flow chart, designing the screen pages models and writing the content of the screen pages ${ }^{[5,7]}$.

System architecture: AVUNET Author allows an instructor to design a pedagogical content for distance education within the virtual University. The goal of this system is to allow the creation of courses that can be used by different instructors. Each instructor can personalize his/her course based on the objectives and problems proper to the concerned course. For that purpose it is recommended to decompose the material in learning units that are independent from each others as much as possible. Each instructor can create his/her course by choosing from a database of learning units the ones that respond to the course's objectives and problems. These learning units are organized in such a way to create a coherent network.

Course representation: AVUNET authoring system is based on a particular pedagogical model related to the notion of conceptual network. This model is the result of a careful thought on how to carry out an interactive course. In fact, the question of how to organize the content of an interactive course is very crucial. The answer to this question leads to the learning unit concept.

One of the basics of the pedagogical method used in this authoring system is the use of a semantic network organizing the learning units with respect to each other. In fact based on a problem or a given objective, the instructor establishes a suitable semantic network. The network once established will appear as a navigation map. This map must be accessible by the learner during his/her training. It visualizes the path followed so far.
A given learning unit is displayed on the main page. Definitions, illustrative examples, simulations and/or experiments clarify it. In some cases theoretical models intended for comprehension of a particular learning unit are available on other pages. The users can active these pages if they choose to do so. From a given learning unit, there is access only to information clarifying $\mathrm{it}^{[7]}$. In this model, each link is connected to an experiment, a definition, an image, etc. which illustrate the learning unit exposed in the initial page.

The cartography of the content can be regarded as a generator of pedagogical documents adapted to the instructors' needs. The principal functions are:

* To skim through the whole course from the starting page.

* To highlight the functional zones with colored links and icons.

* To have the possibility of zooming and filtering.

* To offer a textual and graphical visualization of the course with its properties and statistics.

* To detect and identify graphically the defective local links.

* To visualize the internal and external links.

* To recover the links and the pages.

* To interact with the map to modify or insert a comment on a node or a link.

Other possible uses of the system are to supervise the course architecture changes by comparing its cartographic and to build supports for synthetic courses starting from an initial and supplementing it with individual notes ${ }^{[8-10]}$.

\section{Learner evaluation}

Evaluation role: The evaluation is a fundamental aspect and impossible to circumvent in education. It is indeed crucial that the instructor can evaluate what the students have understood and what they did not. It is also important for the students, during their training, to be able to evaluate their knowledge. There are several ways on how to evaluate a learner. The most used evaluation types are diagnosis evaluation, the formative evaluation, the training evaluation and the summative evaluation $^{[11]}$.

The function of evaluation has an important role within the framework of the training method proposed by the authoring system. In addition to the traditional roles of any evaluation tool, the authoring system evaluation function must make it possible for the learner to distinguish the concepts already learned from those not learned yet. Consequently the facility of spreading out the learning process over several periods of time must be available $e^{[12]}$.

This evaluation thus has double objectives. One it must make it possible for the instructor to propose, at the start of each learning unit, either a pre-test, or a test of pre-requisites. Second it must also makes it possible for learners to self- evaluate at the end of each learning unit by proposing an exit test. 
The various types of possible questions in the evaluation module are as follows: question with simple answer, question with multiple choices, fill in the missing text, make correspondence between two lists, or order a given set of elements.

In this case it is a training evaluation. Actually the self-evaluation has a very important role within the system since it is the learner who decides to be evaluated or not. The instructor makes at the disposal of the learner a questionnaire allowing him/her to check the progress. The evaluation is an integral part of the learning process. Moreover, it is the learner who decides by him/herself whether the obtained result in the evaluation is sufficient to consider the material has been understood.

Learner evaluation system: In AVUNET authoring system, we implemented a module for learner's evaluation and self-evaluation. Only the instructor in charge of the course can access this module in design mode. Each questionnaire is associated with a learning unit. In this mode, the user can create a new questionnaire or open an existing questionnaire. The user must be able to choose whether the questionnaire is intended for the general evaluation or the selfevaluation (should the response time be fixed or not). $\mathrm{He} / \mathrm{she}$ has the possibility of choosing the grading system for each question and for the whole questionnaire (number of correct answers, percentage, grading out 20 , etc.). It is possible to illustrate the question by a text, image and possibly an audio or video file. A feedback is associated to each response in the form of a detailed comment ${ }^{[13]}$.

Once the questionnaire is finished, it is saved on the plate form server. The learner can access via the web navigator or learner interface. The user chooses the questionnaire of the concerned subject. Based on the questionnaire the learner can either take a general evaluation or a self-evaluation. In self-evaluation mode, the user has the choice between having the questions (and even the answers) in order or in a random order. $\mathrm{He} /$ she must be able to choose between displaying the answers instantaneous or wait until the end. The evaluation process is done while moving forward from one question to another with the possibility of returning backward. At the end of the questionnaire, in selfevaluation mode the grade as well as the correct answers and feed back are displayed. In evaluation mode, the results are recorded on the server and/or sent by email to the concerned teacher.

We have designed and implemented a tool to handle the answers given by learners during the automatic evaluation. This tool is used also to analyze the grades of group learners: display learners' lists and their grades, compute the averages, maximum and minimum grade, etc. This option gives the possibility to the learner to compare him/herself to other users automatically. This comparison is also an interesting argument to make it possible for the user to see where does he/she stand comparatively to others ${ }^{[11]}$.

Learner assistant: We developed a learner assistant called AVUNET Navigator to help the user to locate where he/she is with respect to time and space during a navigation session. AVUNET Navigator can be used with any navigator (Netscape Communicator, Internet explorer or other). The principal screen is composed of several windows. Its kernel is made up of two significant modules: a module to extract URL addresses and a module to construct and interact with the navigation map. The second module manages the navigation time. The user has also the possibility to access a glossary containing the terms frequently encountered on Internet and likely to be misunderstood by beginner "surfers". A help window on how to use the software can also be opened.

In order to guarantee the system independence from the navigator, the solution adopted for recovery of the visited addresses consists of an active agent (proxy server). The proxy server is interceded between Web clients and information servers using various protocols, it is used as relay. Each user request is sent, by the software installed on the client side, to the proxy server, which will answer it, directly if it has information in its cash. Otherwise the proxy sends itself the request to the destination server. A copy of each document thus relayed by the proxy server is kept in the cache memory of the proxy (for a variable amount of time). If a document already in the cache is requested from the proxy server, then it is not requested from the distant server. The cache is configured in a way to make it possible to define the cache management according to certain parameters: date of last update of the document, maximum lifespan of the documents in the cache and the time expired since the last use of the document. This system, transparent to the user (except at the time of initial installation), thus offers answers much faster for data available in the cache. It takes more or less the same time, as other system, to get data that is not in its cache. So in general it reduces the traffic over network in a significant way.

The proxy server receives the requests from the navigator, recomposes them if necessary and sends them to the map creation module. The server is installed locally on each user computer to serve as requests. The navigator must be configured to use this proxy. Each request is intercepted and transmitted by the proxy which extracts and preserves necessary information (requested address and elapsed time since the last request) and preserves them. It stores the data in a file, which will be used later on by the map creation module.

Graphical navigation map: The use of graphical map as a device to assist the user navigates the Web is based on a study of the cognitive processes that are involved during the navigation of distributed hypermedia. It 
gives a graphical representation of the conceptual and geographical search path that a user follows in the information space, in agreement with the cognitive navigation maps. The card of navigation, which we conceived is inspired largely from the principles used in the conceptual cards ${ }^{[5,8]}$.

A conceptual map is a mean of representing the existing relations in a set of knowledge as well as the nature of these relations. It is thus a map representing links between concepts of the same topic. It must be able to evolve at the same rate as the acquisition of knowledge by the learner.

The conceptual map can be also a navigation tool, making it possible for a hypertext reader to see the titles of various information units as they are displayed on the screen. The links connecting these units in a form of a traffic network are also displayed. The map is drawn with a goal, within a framework of reference well defined and according to a graphical representation suitable to navigation problem ${ }^{[10]}$.

Map manipulation: In addition to the automatic graphical map generation representing the visited pages, the system allows the drawing of the map from a list of identifiers of pre-selected pages. Also the user can follow the map evolution by creation, deletion of any link or reorganization of the graph, or do only a read of the map for a simple task. All actions that are performed and amount time spent on each page are saved and used for evaluation. This information can be shared among a group of users.

The navigation notion through the web can take another meaning if we make a space correlation between the graph containing the web addresses and a geographic map. The zooming idea starts from here. We propose many zooming levels to make correspondence between different extension of Internet addresses and countries on the geographic map. Different sites having the same extension represent states, pages of the same site represent cities of the same state. This leads to a better comprehension of the Internet hierarchy and helps the user to locate himself within the network. Also the same way we keep track of the time spent during a navigation session can be done for a page, a site or a set of sites with the same extension. The cognitive overload problem is solved by the possibility to do a map zoom (or part of the map) to be able to hide or unhide different details. Also the user can display many graphs corresponding to different zooming level: extension graph, graph of visited sites, graph of pages of the same site, etc.

At any moment it is possible to go backward by clicking on back button. When we are in page mode, we can recognize the complete address and title of a node by simply clicking on it.

Actually to make the comprehension easy a coloring system is adapted. The node colored in green represents the first site (page if we are in page mode) visited by the user. A node colored red represents the last site visited. The intermediate sites are colored orange. If the first site visited is also the last site then it is colored in gray.

The user has also the possibility to save, print or reopen the map constructed during a navigation session. He has also access to the log report during a session that will allow him to do a self-evaluation and to be able to follow his progress during a training period or a search for information. It is also possible to have report indicating the daily interactions and the time spent connected to each site. The graphical map can be used to share information within a group of learners in a cooperative learning environment. Each user can benefit from the experience of the other members of the group $^{[5,10]}$.

Learning and navigation time: According to ${ }^{[14]}$, people do not manage the time the same way while using a media to learn or to just get information. Some users manage their time efficiently but others do not. In this regard people act in different ways. There is a huge difference in the estimation of time spent browsing from one person to another. Certainly our interest in the message being read and our motivation for browsing the Internet play a major role in our time estimation but there are other factors related to the communication tools which also influence our intuition. These observations suggest a dual notion of the time. On one hand each media has its own internal clock, on the other hand the users have their own notion of the time based on the interaction with this media.

Actually there are two kinds of speed (which imply that there are two amount of time for this execution) related to the execution of any phenomena. The first time is the universal time measured by our watches. This is the real time. The duration of the execution of phenomena is equal to the difference between its start and completion times. The second time is the time estimated by the user. This time does not have any mathematical base. It is related to the user's cognitive activity and his perception of the real time.

In order to give to the user the ability to be aware of the time spent during a browsing session, we added panel on which the time is displayed. When the user starts the system he will be asked to enter the expected time to be spent. Once the time expires the system will inform the user by displaying a message. Then the user can decide what to do next. The choices are continue the browsing, save the information find so far, or quit. Actually the user can request the system to display the total time spent on each node. In addition, the user can display the cumulated time spent on each node.

Experiments: In the current version of the authoring system the course creation and learner evaluation modules are working properly. Teacher can use AVUNET Author to design a course based on a preset 
graphical and pedagogical chart. The summary (or table of content) is generated automatically as the instructor is inserting the material. The content classical manipulations are available through the graphical interface bar tools.

The learner evaluation module allows the design of questionnaires for evaluation and self-evaluation. The questionnaires are saved on the plate form server to be used in the learner mode. Also the system can generate the pedagogical bag for the learner. We are working on the implementation and integration of the remaining modules (references management, annotation and marking and graphical manipulation of the content).

We have started testing the current version of the system in real situation within the teaching activities of the instructors. A limited number of instructors (Department of Computer Science, University of Setif, Algeria) have participated in the experiment. The participants showed a real interest in the system. They found out that the graphical interface made the system very easy to use. The integration approach offered by the system was also a big plus. A number of instructors showed a desire to have the possibility of importing data in other formats such as: rtf, txt and html. This functionality is going to be added to the next version.

The tools proposed were designed to answer certain problems. It is necessary to test these tools with the participation of learners. We have tested the developed platform in real practical sessions in cooperation with a teacher and his students. This testing was done in a course about the new information and communication technologies in general and about the web in particular. There were about 100 second year university students, aged from 19 to 22 years. Their major is earth science and all had some experience with some browser. They used the Internet to search for information before. This is a limited experience because we have only the strict minimum needed equipment. Also the connection equipment is not suitable for heavy use.

The experimental environment is made of:

1. Free access to the educational server (self-learning mode) as a complement to the course taken in a regular class.

2. A guided access to the course according to a plan, prepared by the teacher, which is made of a set of documents on the educational server and some links to public documents available on the web.

3. The collection of information about a particular topic from the Internet and the structure of this information into a personnel or group document will be submitted to the teacher using the browsing map.

4. The time panel is used with the browsing map to limit the time of search and access of information. The use of the browsing map has given the students the possibility to structure their knowledge, recognize their limitation and to have a graphical support that can be used to prepare the plan how to search for the needed information. The proxy architecture made it possible; while using the tools, to display on the screen the browser on one window, the sequence of site and the navigation map on another. This solution helps to reduce the cognitive overload of the users.

For the teacher the graphical map can be considered as a tool to analyze the content of what is being taught, to have a better structure of the programs and manuals and to build a plan for the course. The preparation of a guided tour with comments helps to get the new learner to start. These guided tours allow a simple browsing without limiting the freedom of exploring. They include some public documents available over the Internet and some local documents prepared for pedagogical purpose.

\section{CONCLUSION}

AVUNET environment assistants provide an easy access to the available resources and to the various integrated tools. The instructors can create and deploy teaching material in an easy way using this system. It gives the possibility of creating course and related exercises for evaluation or self-evaluation. But it seems that it is necessary at this stage to tackle questions related to the use and the control process that a learner must exert on its own training activity. We plan to design a platform that allows a group of teachers to conceive in a co-operative way intelligent tutoring systems. Also another aims of ours is to evaluate the system in a cooperative training environment either remotely or locally. This evaluation will make it possible to measure the users opinion about the integration of the co-operation and the availability computer aided tools within the framework of training via Internet.

\section{ACKNOWLEDGMENTS}

We would like to thank people who reread for their useful comments and annotations of the first versions of this article, as well as people having brought any contribution to this work and all users and especially anonymous students who were suitable to our observation study and experimentation.

\section{REFERENCES}

1. Douidi, L., 2006. Interfaces et assistances à l'enseignant dans une université virtuelle, thèse de Doctorat en Informatique, Université de Setif, Algéria, Nov.

2. Khentout, C., 2006. Interfaces et assistances à l'apprenant dans une université virtuelle, thèse de Doctorat en Informatique, Université de Setif, Algéria, Nov. 
3. Djoudi, M. and S. Harous, 2002. An environment for cooperative learning over the internet. Intl. Conf. Artificial Intelligence. Las Vegas, USA, pp: 1060-1066.

4. Murray, T., 1998. Authoring knowledge-based Tutors: Tools for content, instructional strategy, student model and interface design. J. Learning Sci, 7: 1 .

5. Lynch, P.J. and S. Horton, 2002. Web Style Guide: Basic Design Principles for Creating Web Sites (2nd Edn.). New Haven, CT: Yale University Press.

6. Shneiderman, B., 1998. Designing the User Interface. Addison Wesley, USA.

7. Wang Baldonado, M.Q. and T. Winograd, 1997. Sense maker: An information exploration interface supporting the contextual evolution of a user's interests. Proc. ACM Conf. Human Factors in Computing Systems, pp: 11-18.

8. Bruley, C. and P. Genoud, 1999. Contribution à une taxonomie des représentations graphiques de l'information. Dixièmes journées francophones sur l'Interaction Homme-Machine. Nantes, France.
9. Earl, M., 2001. Knowledge management strategies: toward a taxonomy. J. Manag. Inform. Syst., 18: 215-233.

10. Miller, S.M. and K.L. Miller, 2000. Theoretical and Practical Considerations in the Design of Webbased Instruction. In B. Abbey (Ed.), Instructional and Cognitive Impacts of Web-based education. Hershey, PA: Idea Group Publishing.

11. Claes, G., 2002. La gestion et l'évaluation pédagogique et administrative par le Web. 2eme Colloque international sur l'université virtuelle. Algiers, Algeria.

12. Born, A.D. and C. Jessup, 2002. An exploration of rubric use in online course assessment. The Forthcoming Proc. Information Resources Management Association (IRMA).

13. Palombra, C. and T. Banta, 1999. Assessment essentials: Planning, implementing and improving assessment in higher education. San Francisco, CA: Jossey Bass.

14. Khentout, C., S. Harous, L. Douidi and M. Djoudi, 2006. Learning and navigation assistance in a hypermedia. Intl. J. Instructional Media, 33: 4. 